\title{
Research metrics of clinical psychology in India: An elephant in the room
}

\author{
Iyer Kamlam Gopalkrishnan ${ }^{1}$, Sam Manickam² \\ ${ }^{1}$ Clinical Child \& Adolescent Neuropsychologist, Oyster Multispecialty Clinic, Thubarahalli, Whitefield, \\ Bengaluru. \\ ${ }^{2}$ Professor of Clinical Psychology, Director - Wellness and Mental Health, Triquetra Technologies, \\ Bengaluru. \\ Corresponding author: Dr. Iyer Kamlam G. \\ Email - iyerkamlam@gmail.com
}

The advent of calculating usage metrics has not been recent. It used to be the manual track of scientific contribution. Later with the foray of modern computers, a shift to measuring digitally the scientific contributions have become feasible, though not easy. From the paper, we have shifted to electronic copies [1]. Virtual access to journals and their articles is a boon to many researchers worldwide. Today, with many research portals offering a virtual platform for researchers worldwide to share, open access to the articles is reasonable assistance [2]. It also makes it easy to assess the other research metrics for an article and the author. For example, ResearchGate $\left(\mathrm{RG}^{\mathrm{TM}}\right)$ generates total "interest" garnered and the other usage metrics of the items posted by the author. The usage metrics are many. The number of online "hits," "reads," "views," "citations," "recommendations" varies in terminologies across different available online platforms. These provide the level of usage of a particular research item [3]. A central issue in science is reads and citations and their relationship [1].

Few domains of science have examined this relationship. Gorraiz et al. [4], in a ten-year publication window period, have analyzed the fields of oncology, computer science, arts and humanities, and economics. Nieder et al. [5] note that few studies have explored the relationship within its subject areas. The present study's authors examine the metrics of a very small subsample of research profiles of clinical psychologists from India.

Using non-random and purposive sampling, five senior researchers' profiles in $\mathrm{RG}^{\mathrm{TM}}$ from the clinical psychology domain in India were accessed on a single given day in the current year (April 18, 2021). The inclusion criteria were that the researcher should be a licensed clinical psychologist (with RCI registration or having trained from Institution/s of national importance). They held a doctoral level of training in psychology / clinical psychology, have experience of 20 years or more, hold positions from government institutions or of national importance, and should have an active profile in $\mathrm{RG}^{\mathrm{TM}}$. We chose only five profiles to gauge the research metrics.

Table 1: Senior Researcher's from Clinical Psychology -- Metrics in RG ${ }^{\mathrm{TM}}$

\begin{tabular}{|c|c|c|c|}
\hline $\begin{array}{c}\text { RG Score } \\
\text { (A) }\end{array}$ & $\begin{array}{c}\text { Number of Citations } \\
\text { (B) }\end{array}$ & $\begin{array}{c}\text { Number of } \\
\text { Views/Reads/Download } \\
\text { s/Hits/Counts } \\
\text { (C) }\end{array}$ & B/C *100 \\
\hline 23.57 & 746 & 118595 & 6.27 \\
\hline 28.94 & 401 & 12634 & 3.17 \\
\hline 30.46 & 238 & 14264 & 1.66 \\
\hline 33.13 & 1424 & 19342 & 7.36 \\
\hline 25.69 & 1231 & 52115 & 2.36 \\
\hline
\end{tabular}

Note: A sample profile of senior researchers' metrics in clinical psychology in India from $R G^{T M}$. $R G^{T M}=$ ResearchGate: $N=5$. 
Table 1 provides a short review of the research metrics. The "reads/views/counts/hits" were high compared to the number of citations received on the articles. A percentage was calculated using the number of reads and citations for each profile. This percentage was obtained by dividing the citations obtained in a given single day by the number of reads/counts on a profile. The rate of citations received by the researchers in clinical psychology was less than $7.5 \%$ overall. This paper seeks to raise the question for future research if the number of citations received is on par with other clinical psychology researchers worldwide. Or if these scores reflect the general trend of all clinical psychology profiles in India? These are questions not satisfied by the small sample size of this study. Nevertheless, it provides us with food-for-thought on the metrics governing citations and reads.

Many controversies surround the metrics of $\mathrm{RG}^{\mathrm{TM}}$ scores [6], which is beyond the scope of this paper. Nevertheless, several western articles have addressed the relationship between reads and citations. Gorraiz et al. [4] found 50 to 140 "downloads" corresponded to one citation but again differed for various domains of science. Nevertheless, the metric of "citations" could depend on the paper's scientific value [7]. The type of articles, such as concept paper, review paper, research report, or research paper, is to be considered in evaluating the usage metrics of citations obtained [5]. These metrics could be well dependent on the journals where the article is published. That is the journal's circulation, peer-reviewing process, the impact factor [8], funding disclosures of the articles [4], to name a few. Many reports on the science behind the usage metrics [9] and if citations accurately reflect the quality of the research articles [10] are well published. Self-citations are also prevalent to increase and promote one's work [11]. Preprints and articles in the press are other newly available metrics that could influence such usage [4] and be further explored. Perneger [12] found that hit counts did predict the number of citations in the subsequent years. And these should be encouraged by the online journals and their respective shared platforms.

Given all the information on the usage metrics, our moot point through this article is to create better usage metrics in terms of citations of our Indian researchers in the domain of clinical psychology. It is challenging to understand the relationship between the reads and citations. A peek into the usage metrics given in Table 1 could be based on pure learning, teaching, information gathering purposes rather than to publish per se [4]. It would be more applicable to domains such as clinical psychology, which is a service-oriented field predominantly.

Therefore, the understanding of how to value contributions is complex. A few recommendations that could put Indian clinical psychology in the research world-map could be the choice of a journal, influencing further subsequent usage metrics such as citation. This relates to good funding as it increases the quality of the research article [7]. More search using engines with Indian open access e-databases of research such as Shodhganga: A reservoir of Indian theses [13] might help in using, citing, and thereby improving citations of our authors. Untapped and unutilized research leads to a considerable wastage of intellectual and financial resources [13].

Additionally, we need to figure out how to optimize our Indian journals and their articles in the search engines for better circulation. This, logically, would mean more collaboration with our researchers. Having review boards for independent researchers of this domain would facilitate good quality and authentic case studies and empirical articles.

These pointers are intended to assist many researchers, independent or affiliated to institutions, to research varied themes and concepts within clinical psychology. It might bring better knowledge to handle issues of research within our subcontinent. To have continued visibility and influence in health settings, we borrow Smith and Thew's [14] observation that the clinical psychologists' stamp as research leaders is essential.

\section{REFERENCES}

1. Kurtz MJ, Bollen J. Usage bibliometrics. Ann Rev Info Sci Tech 2010;44(1):1-64.

2. Wang X, Liu C, Mao W, Fang Z. The open access advantage considering citation, article usage and social media attention. Scientometrics 2015;103(2):555-64.

3. McGillivray B, Astell M. The relationship between usage and citations in an open access mega-journal. Scientometrics 2019;121(2):817-38. 
4. Gorraiz J, Gumpenberger C, Schlögl C. Usage versus citation behaviours in four subject areas. Scientometrics 2014;101(2):1077-95.

5. Nieder C, Dalhaug A, Aandahl G. Correlation between article download and citation figures for highly accessed articles from five open access oncology journals. Springerplus 2013;2(1):261.

6. Kraker P, Lex E. A critical look at the researchgate score as a measure of scientific reputation - Quantifying and analysing scholarly communication on the web (ASCW'15). Available from: http://ascw.knowcenter.tugraz.at/2015/05/26/kraker-lex-a-critical-look-at-the-researchgate-score/

7. Lee KP. Association of journal quality indicators with methodological quality of clinical research articles. JAMA 2002;287(21):2805.

8. Gallagher EJ, Barnaby DP. Evidence of methodologic bias in the derivation of the science citation index impact factor. Ann Emerg Med 1998;(1):83-6.

9. Seglen PO. The skewness of science. J Am Soc Inf Sci 1992;43(9):628-38.

10. Opthof T. Sense and nonsense about the impact factor. Cardiovasc Res 1997;33(1):1-7.

11. Van Noorden R, Singh Chawla D. Hundreds of extreme self-citing scientists revealed in new database. Nature 2019;572(7771):578-9.

12. Perneger TV. Relation between online "hit counts" and subsequent citations: prospective study of research papers in the BMJ. BMJ 2004;329(7465):546-7.

13. Shodhganga : a reservoir of Indian theses @ INFLIBNET [Internet]. [cited 2021 Oct 16]. Available from: https://shodhganga.inflibnet.ac.in/

14. Smith KV, Thew GR. Conducting research in clinical psychology practice: Barriers, facilitators, and recommendations. Br J Clin Psychol 2017;56(3):347-56.

$* * * * * * * * * * * * * * * * * * * * * * * * * * * * * * * *$

Acknowledgements - Nil

Conflict of Interest - Nil

Funding - Nil 